\title{
Titanium mesh implants exposure after cranioplasty in two children: involvement of osteogenesis?
}

\author{
Han-song Sheng ${ }^{1,3+}$, Fang Shen ${ }^{2 \dagger}$, Mao-de Wang ${ }^{3}$, Jian Lin ${ }^{1}$, Fen-chun Lin ${ }^{1}$, Bo Yin ${ }^{1}$ and Nu Zhang ${ }^{1 *}$
}

\begin{abstract}
Background: Although technically regarded as a simple procedure, titanium mesh cranioplasty could lead to various surgical complications, including postoperative implant exposure. However, there is little data available on the occurrence and risk factors of this complication in the pediatric population.

Cases presentation: Two pediatric male patients, one 12-year-old and one 7-year-old, had decompressive craniectomy after traumatic brain injuries and subsequent cranioplasty with titanium mesh. However, both patients had skin defects developed gradually at the scalp adjacent to the surgical incisions, 11 and 7 months after cranioplasty, respectively. Implants removal surgeries were then delivered and, during the operation, some bone debris were found just beneath the skin defects in both patients. Because microbiological culture results of the exudations were negative, in addition to the long interval between cranioplasty and developments of skin defects, surgical infections might not be major causes of the observed titanium implants exposures. On the other hand, local osteogenesis and impaired scalp blood supply might contribute to their occurrence.
\end{abstract}

Conclusions: Efforts should be made to achieve complete clearance of bone debris and protect scalp blood supply during the initial decompressive craniectomy in order to minimize the risks of subsequent titanium mesh exposures.

Keywords: Cranioplasty, Titanium mesh, Complications, Osteogenesis, Children

\section{Background}

Decompressive craniectomy is a routine neurosurgical procedure used to treat patients with refractory high intracranial pressures caused by traumatic brain injury, stroke, and other vascular and neoplastic conditions $[1,2]$. The increased survival rates of trauma patients who underwent decompressive craniectomy necessitate reconstructions of these cranial defects for both cosmetic and protective purposes [3]. In addition, cranioplasty is also reported to produce cognitive benefits in these patients, especially in children [4]. Various materials, such as autogenous/xenogenous bone grafts, gold and polymethylmethacrylate, have been used for cranioplasty. However,

\footnotetext{
*Correspondence: wzneurosurgery@outlook.com

${ }^{\dagger}$ Equal contributors

${ }^{1}$ Department of Neurosurgery, Second Affiliated Hospital of Wenzhou Medical University, 109 Xueyuan Western Road, Wenzhou, Zhejiang 325027, China

Full list of author information is available at the end of the article
}

there is a general preference of titanium mesh to repair the cranial defects because of its biocompatibility, rigidity, lightness, as well as compatibility with magnetic resonance imaging scans [5]. Although regarded as a surgically simple procedure, cranioplasty with titanium mesh has been associated with a high surgical complication rate that is up to $34 \%$ [6]. Among them, surgical infection and postoperative hematoma usually require reoperation $[7,8]$.

Pediatric patients differ from their adult counterparts both physiologically and anatomically. For example, regrowth of the cranium is more common in children than in adults [9]. However, when autologous bone is used for cranioplasty, the pediatric population is reported to have a much higher bone absorption rate than adults [10]. There is still little data available regarding complications associated with titanium cranioplasty in pediatric patients and the associated risk factors [11], and we reported here two pediatric cases of titanium mesh exposure after cranioplasty. The key findings of both cases 
are development of osteogenesis just beneath the skin defects and long interval between cranioplasty and mesh exposure. Therefore, instead of surgical infection, osteogenesis of the remaining bone debris and impaired scalp blood supply might contribute to the occurrences of the postoperative mesh exposure.

\section{Case presentation}

The first case was a 12-year-old male patient who complained about a scalp defect $(2.0 \times 3.0 \mathrm{~cm})$ and exposure of the titanium mesh underneath. On review of his medical history, he received a right side decompressive craniectomy because of traumatic brain injury. During the initial emergence decompression procedure, a right fronto-parieto-temporal bone flap $(11.0 \times 13.0 \mathrm{~cm})$ was removed and, in order to reduce adhesions between the cortex/dura and the overlying temporalis muscle/galea, an artificial dura mater was used for dura repair (Fig. 1a). Three months after the initial operation, threedimensional (3D) computed tomography (CT) reconstruction of the cranium revealed the existence of some small residual bone debris at the surgical site under the scalp (Fig. 1b\&c). These CT images were also used for manufacturing of the titanium mesh. For cranioplasty, routine perioperative antibiotics and post-operative subgaleal drainage were used. The postoperative course was uneventful and follow-up CT scans performed 2 months (Fig. 1d\&e), 5 months (Fig. 1f) and 9 months (Fig. 1g) after titanium cranioplasty revealed gradual enlargements of the bone debris under the implant. Nine months after cranioplasty, the patient complained about thinning and reddening of the right fronto-parietal scalp but no treatment was given at that time. Eleven months after cranioplasty, a scalp defect $(2.0 \times 3.0 \mathrm{~cm})$ appeared at the right parietal scalp with exposure of the titanium mesh underneath (Fig. 1h). Local sterile dressing was first given for several days and, in view of no sign of spontaneous healing of the defect, an implant removal surgery was delivered. During the operation, we found the implant under the skin defect was covered by granulation tissue $(5.0 \times 6.0 \mathrm{~cm})$ and, in accordance with preoperative CT findings, a small bone debris $(1.0 \times 2.0 \mathrm{~cm})$ was found under the mesh, right under the skin defect (Fig. 1i\&j). Granulation tissues and exudations were
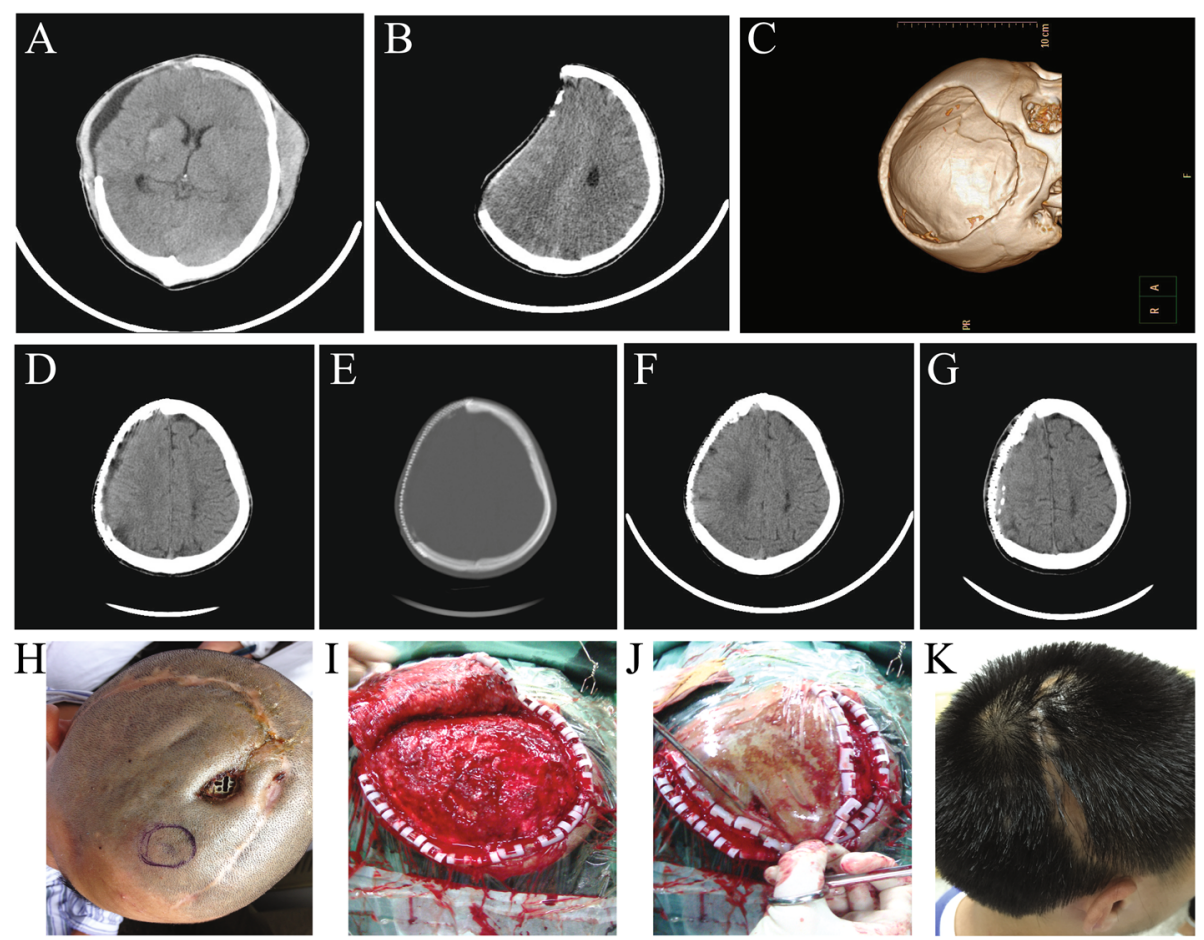

Fig. 1 Cranial computed tomography (CT) scan of a 12-year-old male patient who undergone a right decompressive craniectomy because of traumatic brain injury (a). Three months after the initial operation, $C T$ scan $(\mathbf{b})$ and 3D reconstruction of the cranium (c) revealed the existence of residual bone scraps under the scalp (white arrow). Follow-up CT scans after cranioplasty with titanium mesh at 2 months (d \& e), 5 months (f) and 9 months $(\mathbf{g})$ indicated gradually enlargement of the bone debris under the implant (white arrow). Eleven months after cranioplasty, a scalp defect $(2.0 \times 3.0 \mathrm{~cm}$, white arrow head) appeared at the right parietal skin with exposure of the titanium mesh underneath (h). During the implant removal operation, we found the implant under the skin defect was covered by granulation tissue $(5.0 \times 6.0 \mathrm{~cm}$, multiple white arrow) and an enlarged bone debris $(1.0 \times 2.0 \mathrm{~cm}$, single white arrow) was found under the mesh (i). After removal of the titanium mesh, the ossification site (white arrow) was found to be right under the defect (white arrow head) (j). All the bone debris were removed during operation (e). Clinical follow-up at 2 months later showed satisfactory wound healing $(\mathbf{k})$ 
sampled and sent for microbiological cultures. However, negative result was reported. After removal of the implant, and clear of all the granulation tissues and bone debris, the incision was closed in layers with sutures. The patient received routine perioperative antibiotic treatments and was discharged without complication 2 weeks after the operation. Clinical follow-up at 2 months later showed satisfactory wound healing (Fig. 1k).

The second case was a 7-year-old male patient admitted to our hospital for similar reason. His scalp defect was $1.0 \times 2.0 \mathrm{~cm}$ and he had received a bifrontal decompressive craniectomy because of traumatic brain injury. Seven months after the initial operation, a second cranioplasty was arranged to fix the cranial defects with preformed titanium mesh. The postoperative course was uneventful and the patient was discharged from the hospital. Seven months after cranioplasty, the patient complained about the development of a small skin defect $(1.0 \times 2.0 \mathrm{~cm})$ at the right frontal scalp, adjacent to the incision (Fig. 2a). Initial treatments included sterile dressing and intravenous injection of antibiotics. However, because the defect gradually become larger, implant removal was performed (Fig. 2b). Similar to the first case, the implant under the defect was covered by granulation tissue $(4.0 \times 5.0 \mathrm{~cm})$ and distinctive ossifications $(0.5 \times 1.0 \mathrm{~cm})$ were found under the mesh, just beneath the defect (Fig. 2c-e). Specimens were taken and negative microbiological cultures results were reported. After removal of the implant and debridement, the incision was closed with sutures. This patient also received empirical antibiotic treatment after the operation and was discharged without complication. Clinical follow-up at 5 months later showed satisfactory wound healing (Fig. 2f).

\section{Discussion}

In order to restore the skull defect produced by craniectomy, cranioplasty is usually performed with either autogenous or alloplastic materials [12]. Although autogenous bone graft is still the best choice whenever possible [13], titanium mesh has been a popular alternative in view of its lightweight, rigidity, and biocompatibility [14]. Although regarded as a technically simple procedure, cranioplasty has been suggested to have a high complication rate. For example, scalp infections and exposure of the implant may sometime be life threatening and usually require implant removal. However, surgical infections are not likely to be the major causes of implants exposures in our cases. First, neither the patients' symptoms, e.g. body temperatures, nor the lab tests, e.g. microbiological cultures results, supported the existence of bacterial infections. Second, the time interval between cranioplasty and development of scalp defects was much longer than that were reported in

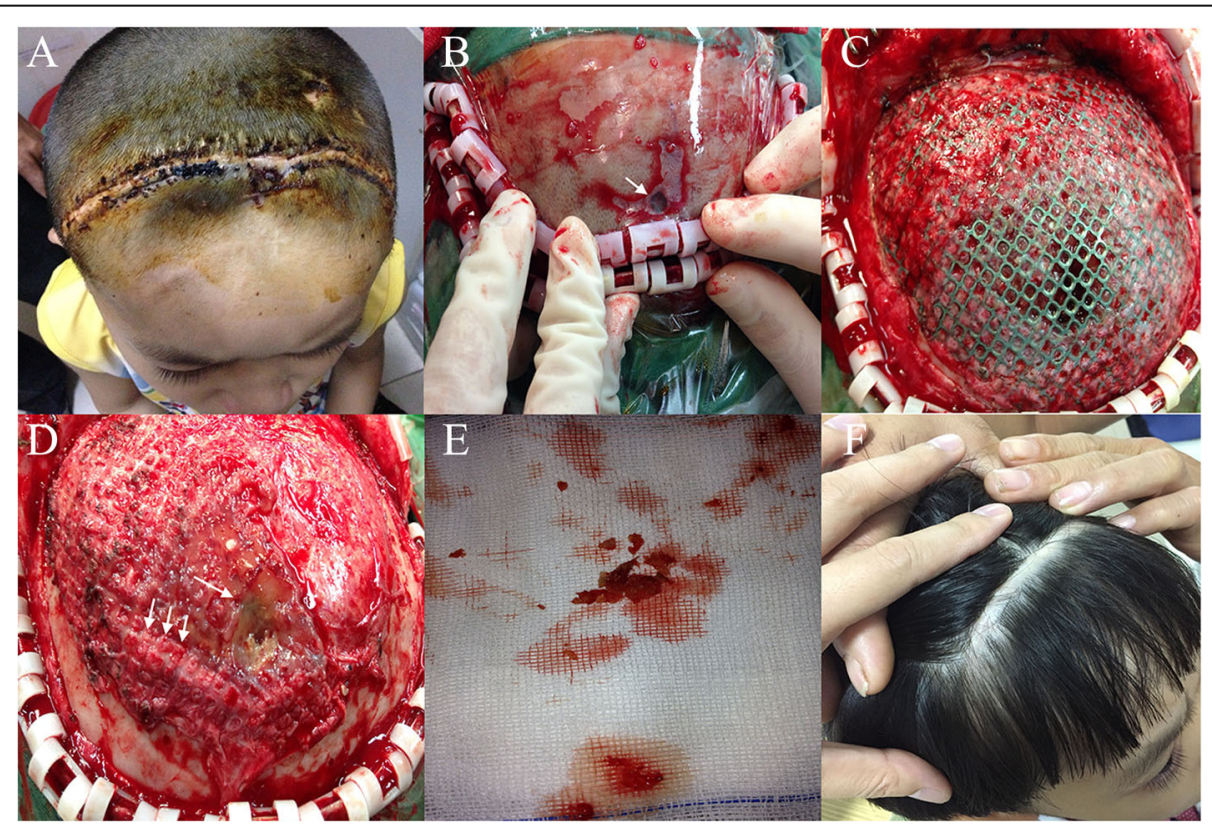

Fig. 2 A 7-year-old male patient who had undergone a bifrontal decompressive craniectomy presented with scalp defect $(0.5 \times 1.0 \mathrm{~cm}$, white arrow head) at right frontal scalp 7 months after titanium mesh cranioplasty $(\mathbf{a})$. Because of gradual increases in the defect size $(1.0 \times 2.0 \mathrm{~cm}$, white arrow), an implant removal operation was performed 7 months after cranioplasty (b). During the operation, the implant under the skin defect was covered by granulation tissue $(4.0 \times 5.0 \mathrm{~cm}$ in size, multiple white arrows) and ossifications $(0.5 \times 1.0 \mathrm{~cm}$, single white arrow) was located right under the defect $(\mathbf{c} \& \mathbf{d})$. All the bone debris were removed during operation $(\mathbf{e})$. Clinical follow-up at 5 months later showed satisfactory wound healing ( $f$ ) 
literatures. For example, previous studies have shown the median time from implantation to removal of the implant after infection was 55 days [5]. In our both cases, the titanium mesh was exposed 11 and 7 months after cranioplasty. On the other hand, osteogenesis from the residual bone debris might, at least partially, be responsible for the occurrence of scalp defect. This hypothesis is based on intraoperative observation of a relatively large area of granulation tissue and, more importantly, an enlarged bone debris just under the defects. Previous study has also suggested titanium mesh, as an alloplastic material, is favorable for osteogenesis under the implant [15]. In addition, both cases were pediatric patients and children have greater capacity for tissue regeneration, including ossification of the skull defects [16]. The possible explanation for this osteogenesis related implant exposure might be as flows. First, osteogenesis is a multistep process that involves inflammatory response and the various inflammatory cytokines released during the reossification process might influence adjacent skin healing. Second, the gradually enlarged bone debris might also impart a mechanic stress on the adjacent skin. Therefore, both chemical and physical factors might contribute to the development of scalp defect. Since these residual bone scraps might be a risk factor for development of scalp defect, cautions should be taken to debride the wound completely before closure of the skin incision at the initial craniectomy, especially in the pediatric patients. Other factors, such as impaired blood supply to the scalp, might also contribute to the development of defect and subsequent implant exposure. Further clinical and laboratory studies are needed to clarify the relationship between osteogenesis and development of scalp defects in pediatric patients who received titanium mesh cranioplasty.

\section{Conclusions}

Osteogenesis and impaired blood supply might contribute to the development of scalp defects and exposure of titanium mesh underneath in pediatric patients. Complete debridement of bone scraps and protection of the blood supply at the initial craniectomy might help to reduce the risk of its occurrence.

\section{Abbreviations}

3D: Three-dimensional; CT: Computed tomography

\section{Acknowledgements}

Not applicable.

Funding

This work was supported by Science Technology Department of Zhejiang Province (Grant No. 2016C33SA300055 \& 2015C33144).

Availability of data and materials

Data sharing not applicable to this article as no datasets were generated or analyzed during the current study.

\section{Authors' contributions}

HSS \& FS conceived \& wrote the manuscript. MDW, JL, FCL, BY did data collection and contributed to manuscript writing. NZ did review and final approval of manuscript. All authors read and approved the final manuscript.

\section{Competing interests}

The authors declare that they have no competing interests.

\section{Consent for publication}

Written informed consent regarding the publication of this case report and its accompanying images was obtained from the patients' parents. Copies of the written consent are available for review upon request.

\section{Ethics approval and consent to participate}

Consents to conduct and report of this study was obtained from the Ethics Committee of Second Affiliated Hospital of Wenzhou Medical University.

\section{Author details}

'Department of Neurosurgery, Second Affiliated Hospital of Wenzhou Medical University, 109 Xueyuan Western Road, Wenzhou, Zhejiang 325027, China. ${ }^{2}$ Department of Neurosurgery, Ningbo No.2 Hospital, Ningbo, China. ${ }^{3}$ Department of Neurosurgery, First Affiliated Hospital of Medical College of Xi'an Jiaotong University, Xi'an, China.

Received: 24 July 2016 Accepted: 10 January 2017

Published online: 05 April 2017

\section{References}

1. Sahuquillo J and Arikan F. Decompressive craniectomy for the treatment of refractory high intracranial pressure in traumatic brain injury. Cochrane Database Syst Rev. 2006: CD003983. doi:10.1002/14651858.CD003983.pub2.

2. Vahedi $\mathrm{K}$, Hofmeijer J, Juettler $\mathrm{E}$, et al. Early decompressive surgery in malignant infarction of the middle cerebral artery: a pooled analysis of three randomised controlled trials. Lancet Neurol. 2007;6:215-22.

3. Chang V, Hartzfeld P, Langlois M, Mahmood A, Seyfried D. Outcomes of cranial repair after craniectomy. J Neurosurg. 2010;112:1120-4.

4. Winkler PA, Stummer W, Linke R, Krishnan KG, Tatsch K. Influence of cranioplasty on postural blood flow regulation, cerebrovascular reserve capacity, and cerebral glucose metabolism. J Neurosurg. 2000;93:53-61.

5. Hill CS, Luoma AM, Wilson SR, Kitchen N. Titanium cranioplasty and the prediction of complications. Br J Neurosurg. 2012;26:832-7.

6. Mukherjee S, Thakur B, Haq I, Hettige S, Martin AJ. Complications of titanium cranioplasty-a retrospective analysis of 174 patients. Acta Neurochir. 2014:156:989-98. discussion 98.

7. Kim H, Sung SO, Kim SJ, Kim SR, Park IS, Jo KW. Analysis of the factors affecting graft infection after cranioplasty. Acta Neurochir. 2013;155:2171-6.

8. Cheng YK, Weng HH, Yang JT, Lee MH, Wang TC, Chang CN. Factors affecting graft infection after cranioplasty. J Clin Neurosci. 2008;15:1115-9.

9. Piedra MP, Thompson EM, Selden NR, Ragel BT, Guillaume DJ. Optimal timing of autologous cranioplasty after decompressive craniectomy in children. J Neurosurg Pediatr. 2012;10:268-72.

10. Rocque BG, Amancherla K, Lew SM, Lam S. Outcomes of cranioplasty following decompressive craniectomy in the pediatric population. J Neurosurg Pediatr. 2013;12:120-5.

11. Bowers CA, Riva-Cambrin J, Hertzler 2nd DA, Walker ML. Risk factors and rates of bone flap resorption in pediatric patients after decompressive craniectomy for traumatic brain injury. J Neurosurg Pediatr. 2013;11:526-32.

12. Cabraja M, Klein M, Lehmann TN. Long-term results following titanium cranioplasty of large skull defects. Neurosurg Focus. 2009;26:E10.

13. Grant GA, Jolley M, Ellenbogen RG, Roberts TS, Gruss JR, Loeser JD. Failure of autologous bone-assisted cranioplasty following decompressive craniectomy in children and adolescents. J Neurosurg. 2004;100:163-8.

14. Wiggins A, Austerberry R, Morrison D, Ho KM, Honeybul S. Cranioplasty with custom-made titanium plates-14 years experience. Neurosurgery. 2013;72: 248-56. discussion 56.

15. Gundeslioglu OA, Ince B. Exposed titanium mesh and dura persisting for 8 years after cranioplasty. J Craniofacial Surg. 2013;24:655-6.

16. Josan VA, Sgouros S, Walsh AR, Dover MS, Nishikawa H, Hockley AD. Cranioplasty in children. Childs Nerv Syst. 2005;21:200-4. 